\title{
Técnica de mosaicoplastia no tratamento de lesões osteocondrais isoladas do côndilo femoral do joelho - estudo retrospectivo*
}

\section{Mosaicplasty Technique in the Treatment of Isolated Knee Femoral Condyle Osteochondral Lesions - a Retrospective Study}

\author{
Samir Karmali ${ }^{1}$ Rui Guerreiro ${ }^{1}$ Daniel Sá da Costa ${ }^{1}$ Jorge Fonseca ${ }^{1} \quad$ Ricardo Gonçalves ${ }^{20}$ \\ ${ }^{1}$ Departamento Ortopédico, Hospital Vila Franca de Xira, Vila Franca \\ de Xira, Portugal \\ 2 Departamento Ortopédico, Unidade de Ombro e Cotovelo, Hospital \\ Privado de Gaia, Vila Nova de Gaia, Portugal \\ Address for correspondence Ricardo Gonçalves, MD, Departamento \\ Ortopédico, Unidade de Ombro e Cotovelo, Hospital Privado de Gaia, \\ Rua Fernão de Magalhães, $n^{\circ}$ 2, Fração E, 24 4404-501, Vila Nova de \\ Gaia, Portugal (e-mail: goncalves.ricardo@gmail.com).
}

Rev Bras Ortop 2019;54:316-321.

\begin{abstract}
Resumo
Palavras-chave

- osteocondrite/ diagnóstico

- osteocondrite/ cirurgia

- artroscopia

- articulação do joelho

- cartilagem articular

Objetivo Lesões osteocondrais focais do joelho são encontradas em dois terços dos pacientes submetidos a artroscopia; seu tratamento, quando isoladas e, principalmente, em indivíduos jovens, ainda é debatido. O presente estudo analisa os resultados obtidos com a aplicação da técnica de mosaicoplastia no tratamento de lesões osteocondrais isoladas do côndilo femoral do joelho.

Métodos Estudo retrospectivo de pacientes submetidos à mosaicoplastia e análise subjetiva com pontuações do International Knee Documentation Committee (IKDC, na sigla em inglês) antes e após a cirurgia.

Resultados Um total de 13 casos, com média de idade de 34 anos; pacientes do sexo masculino $(n=4 ; 31 \%)$ apresentaram média de idade de 23 anos (17-31 anos), e pacientes do sexo feminino $(n=9 ; 69 \%)$ apresentaram média de 39 anos; (16-56 anos); femoral medial ou lateral ( $n=11,85 \%$ versus $n=2,15 \%$, respectivamente); o tamanho médio da lesão foi de $1,8 \mathrm{~cm}^{2}(0,6-4 \mathrm{~cm})$; o tempo médio de acompanhamento foi de $5,045 \pm 3,47$ anos (1,15-11,01 anos). A pontuação IKDC média préoperatória foi 31,63 pontos ( $\pm 20,24)$, e a pós-operatória foi 74,18 pontos $( \pm 20,26)$. A diferença entre as pontuações IKDC obtidas depois e antes da cirurgia foi de 42,55 ( $\pm 21,05$ ) pontos, com o aumento mínimo de 8,1 pontos e o aumento máximo de 82,8 pontos. Uma diferença estatística significativa $(p<0,001)$ foi encontrada entre a pontuação IKDC antes e após a cirurgia. Uma relação estatisticamente significativa $(p=0,038)$ foi observada entre o aumento da pontuação IKDC (a diferença entre a pontuação pré- e pós-operatória) e as dimensões da lesão.
\end{abstract}

\footnotetext{
O presente estudo foi conduzido no Departamento Ortopédico do Hospital Vila Franca de Xira, Portugal.

(D) Ricardo Gonçalves's ORCID is https://orcid.org/0000-0002-03117023.
}

received

January 7, 2018

accepted

May 28, 2018
DOI https://doi.org/

10.1055/s-0039-1691763.

ISSN 0102-3616.
Copyright $(2019$ by Sociedade Brasileira License terms de Ortopedia e Traumatologia. Published by Thieme Revnter Publicações Ltda, Rio de Janeiro, Brazil 


\begin{abstract}
Keywords

- osteochondritis/ diagnosis

- osteochondritis/ surgery

- arthroscopy

- knee joint

- cartilage, articular

Objective Focal osteochondral lesions of the knee are found in two thirds of patients undergoing arthroscopy; their treatment, when isolated and especially in young individuals, remains a debating topic. The present study analyzes the results obtained by the application of the mosaicplasty technique on the treatment of isolated knee femoral condyle osteochondral lesions.

Methods Retrospective study of patients submitted to mosaicplasty and to subjective analyses with pre- and postsurgery International Knee Documentation Committee (IKDC) scores.

Results A total of 13 cases with an average age of 34 years old, with male patients ( $n=4 ; 31 \%$ ) with an average age of 23 years old (range: $17-31$ years old), and female patients ( $n=9 ; 69 \%$ ) with an average age of 39 years old (range: $16-56$ years old); medial versus lateral femoral $(n=11 ; 85 \%$ versus $n=2 ; 15 \%)$; the average size of the lesion was $1.8 \mathrm{~cm}^{2}$ (range: $0.6-4 \mathrm{~cm}^{2}$ ); average follow-up time: $5.045 \pm 3.47$ years (range: $1.15-11.01$ years). The average preoperative IKDC score was of 31.63 points ( \pm 20.24$)$, the average postoperative IKDC score was of 74.18 points $( \pm 20.26)$. The difference between the post- and preoperative IKDC scores was of 42.55 points ( \pm 21.05 ), being the minimal score increase of 8.1 points and the maximum score increase of 82.8 points. A statistically significant difference $(p<0.001)$ was found between the IKDC scores before and after the surgery. A statistically significant relation ( $p=0.038$ ) was found between the IKDC score increase (the difference between the postoperative and the preoperative scores) and the dimension of the lesion.

Conclusions Mosaicplasty with osteochondral autograft transfer, when adequately used, can produce excellent results with great durability and functional impact, low morbidity rates and costs. Expansion of the indication criteria shows promising midterm and long-term results.
\end{abstract}

Conclusões A mosaicoplastia com transferência de autoenxerto osteocondral, quando adequadamente usada, pode produzir resultados excelentes com grande durabilidade e impacto funcional, baixas taxas de morbidade e baixos custos. A expansão dos critérios de indicação mostra resultados promissores no médio e longo prazo.

\section{Introdução}

Lesões osteocondrais focais do joelho são encontradas em dois terços dos pacientes submetidos a artroscopia. ${ }^{1}$ o tratamento destas lesões, quando isoladas e, principalmente, em indivíduos jovens, ainda é debatido. ${ }^{2-4} \mathrm{~A}$ artroplastia do joelho não é uma boa solução, e ainda não há uma técnica que possa restituir, ad integrum, a superfície articular da cartilagem hialina normal. ${ }^{2,4-6}$ Hoje, as técnicas existentes seguem princípios paliativos (desbridamento e condroplastia), reparadores (microfratura) e restauradores, como o implante de condrócitos autólogos (ACI, na sigla em inglês), a transferência de autoenxerto osteocondral (OTA, na sigla em inglês) e o aloenxerto osteocondral (OCA, na sigla em inglês). ${ }^{2,3}$ A OTA, na qual podemos incluir a mosaicoplastia, tem sido aplicada principalmente para alívio sintomático e para a tentativa de recuperação da superfície articular com propriedades biofuncionais semelhantes aos de uma articulação danificada, visando a preservação da função e a redução da progressão da doença osteoarticular. ${ }^{2,7-9}$ O presente estudo tem por objetivo analisar os resultados obtidos com a aplicação da técnica de mosaicoplastia no tratamento das lesões osteocondrais do côndilo femoral do joelho.

\section{Metodologia}

Análises retrospectivas de pacientes com lesões osteocondrais do joelho, selecionados e submetidos à técnica de restauração denominada mosaicoplastia, na nossa instituição entre 2001 e 2015. Os critérios de inclusão foram: pacientes com idade $\leq 60$ anos, com lesão osteocondral única do côndilo femoral (Outerbrige III/IV) $<4 \mathrm{~cm}^{2}$ na ressonância magnética (RM) pré-operatória (International Cartilage Repair Society [ICRS]) e confirmada durante a artroscopia e com acompanhamento de pelo menos 1 ano. Pacientes com lesões associadas (por exemplo, na tíbia ou na patela), mau alinhamento, ou previamente submetidos a cirurgia de realinhamento, foram excluídos (-Tabela 1).

O procedimento foi realizado utilizando o Sistema Osteocondral de Transferência de Autoenxerto (Osteochondral Autograft Transfer System [OATS]) (Arthrex, Inc., Naples, 
Tabela 1 Critérios de inclusão e exclusão

\begin{tabular}{|l|}
\hline Critérios de inclusão \\
\hline Idade $\geq 16$ anos e $\leq 60$ anos \\
\hline Lesão osteocondral isolada no côndilo femoral \\
\hline Grau III/IV (Outerbrigde) \\
\hline Tamanho $\leq 4 \mathrm{~cm}^{2}$ \\
\hline Acompanhamento $>1$ ano \\
\hline Critérios de exclusão \\
\hline Lesões associadas (por exemplo, tíbia ou patela) \\
\hline $\begin{array}{l}\text { Mau alinhamento ou realização prévia de cirurgia de } \\
\text { realinhamento }\end{array}$ \\
\hline Instabilidade ligamentar \\
\hline Doença inflamatória \\
\hline
\end{tabular}

FL, USA) e de acordo com a técnica descrita por Hangody ${ }^{7}$, através de artroscopia com miniartrotomia para a extração e a transferência do enxerto da sua localização (região troclear, zona de não carga) para o defeito osteocondral (-Fig. 1). 0 protocolo pós-operatório foi composto por restrição de carga durante por 2 semanas, seguida de carga parcial (10-20\% do peso corporal) até a $6^{\underline{a}}$ semana, carga total até 6 meses e, posteriormente, atividade irrestrita.

Os pacientes foram submetidos a avaliação subjetiva com o formulário do International Knee Documentation Committee (IKDC, na sigla em inglês) no período entre a lesão e a cirurgia e, novamente, 1 ano após a cirurgia. A análise dos dados foi feita com o programa IBM SPSS Statistics for Windows, Versão 22.0 (IBM Corp., Armonk, NY, USA).

\section{Resultados}

A partir das análises dos 13 casos elegíveis para o estudo (-Tabela 2), encontramos uma média de idade de 34 anos; pacientes do sexo masculino $(n=4 ; 31 \%)$ apresentaram uma média de 23 anos (17-31 anos), e pacientes do sexo feminino ( $n=9 ; 69 \%)$ apresentaram uma média de 39 anos (16-56 anos). As lesões afetaram mais o côndilo femoral medial em comparação com o lateral ( $n=11 ; 85 \%$ versus $n=2 ; 15 \%)$ e o tamanho médio da lesão foi de $1,8 \mathrm{~cm}^{2}(0,6-$ $4 \mathrm{~cm}$ ), com 7 lesões $>2 \mathrm{~cm}^{2}$. 0 tempo médio de acompanhamento dos pacientes foi de 5,045 $\pm 3,47$ anos $(1,15-$ 11,01 anos). Durante o $1^{\circ}$ ano, nenhum dos pacientes desenvolveu complicações infecciosas ou do local doador; 1 paciente ( 1 mulher de 54 anos) foi submetido a
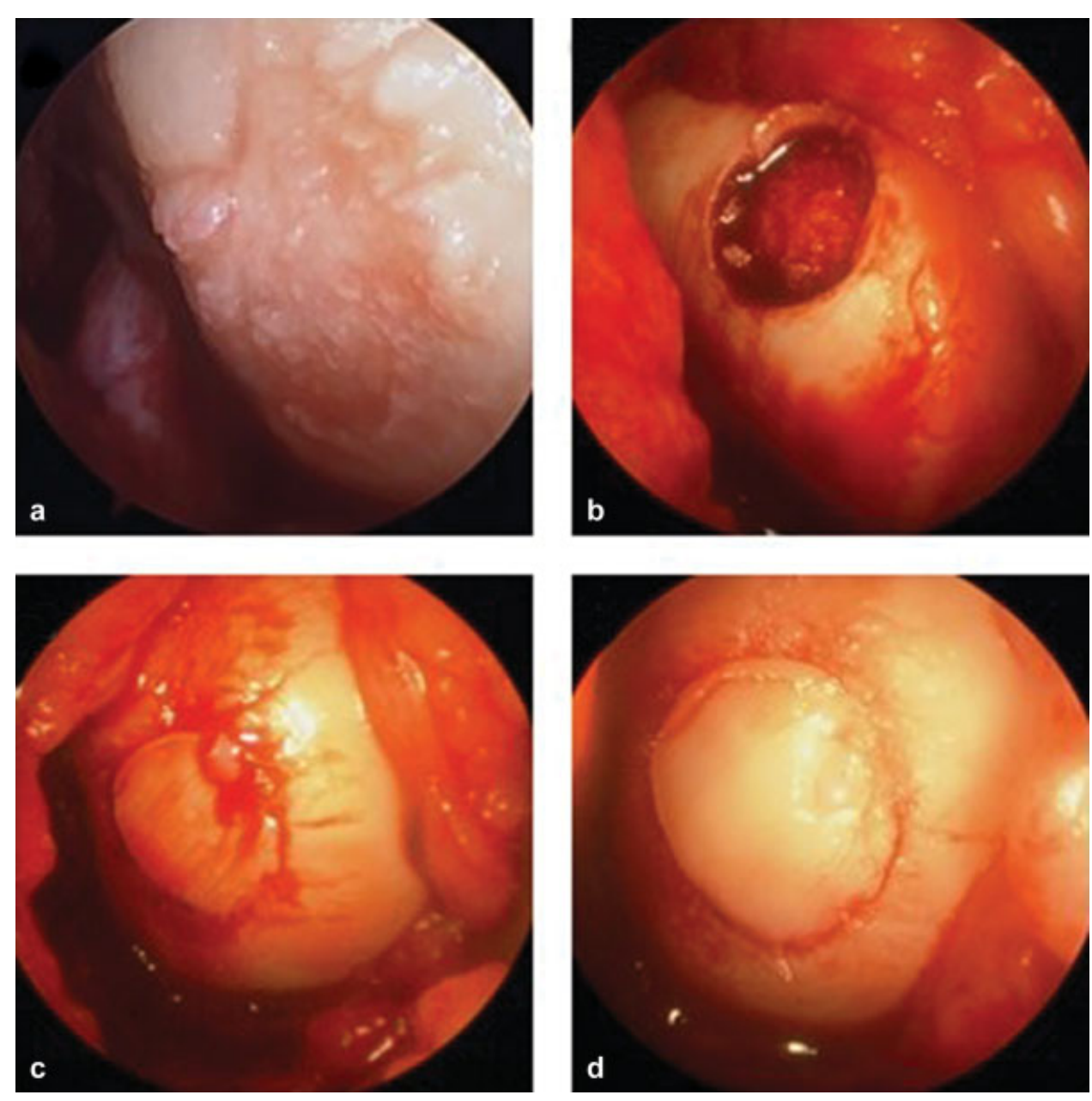

Fig. 1 Técnica cirúrgica: (a) lesão osteocondral do côndilo femoral medial; (b) área doadora - área troclear circunferencial sem sustentação de peso; (c) e (d) reparo final. 
Tabela 2 Pacientes incluídos no grupo de estudo

\begin{tabular}{|l|l|l|l|l|l|l|l|}
\hline $\mathbf{n}$ & $\begin{array}{l}\text { Idade } \\
\text { (anos) }\end{array}$ & Gênero* $^{*}$ & Localização** $^{* *}$ & $\begin{array}{l}\text { Tamanho } \\
\left(\mathbf{c m}^{2}\right)\end{array}$ & IKDC pré-operatório & IKDC pós-operatório & $\begin{array}{l}\text { Acompanhamento } \\
\text { (anos) }\end{array}$ \\
\hline 1 & 25 & M & MFC & 1 & 79,3 & 100 & 3,00 \\
\hline 2 & 16 & F & MFC & 3 & 46 & 90,8 & 11,01 \\
\hline 3 & 16 & F & LFC & 4 & 36,8 & 88,5 & 11,01 \\
\hline 4 & 17 & M & MFC & 2 & 3,3 & 44,8 & 8,01 \\
\hline 5 & 54 & F & MFC & 1 & 54 & 62,1 & 6,79 \\
\hline 6 & 18 & M & MFC & 3 & 18,4 & 92 & 4,00 \\
\hline 7 & 36 & F & MFC & 2 & 17,2 & 100 & 2,00 \\
\hline 8 & 30 & F & LFC & 1 & 40,2 & 85,1 & 3,00 \\
\hline 9 & 42 & F & MFC & 0,6 & 17,2 & 44,8 & 2,36 \\
\hline 10 & 48 & F & MFC & 2 & 12,6 & 72,4 & 1,15 \\
\hline 11 & 56 & F & MFC & 0,9 & 27,6 & 50,6 & 2,78 \\
\hline 12 & 31 & M & MFC & 1 & 34,5 & 74,7 & 7,97 \\
\hline 13 & 53 & F & MFC & 2 & 24,1 & 58,6 & 2,51 \\
\hline
\end{tabular}

Abreviaturas: F, feminino; IKDC, International Knee Documentation Committee; LFC, côndilo femoral lateral; M, masculino; MFC, côndilo femoral medial. *gênero,

${ }^{* *}$ Iocalização.

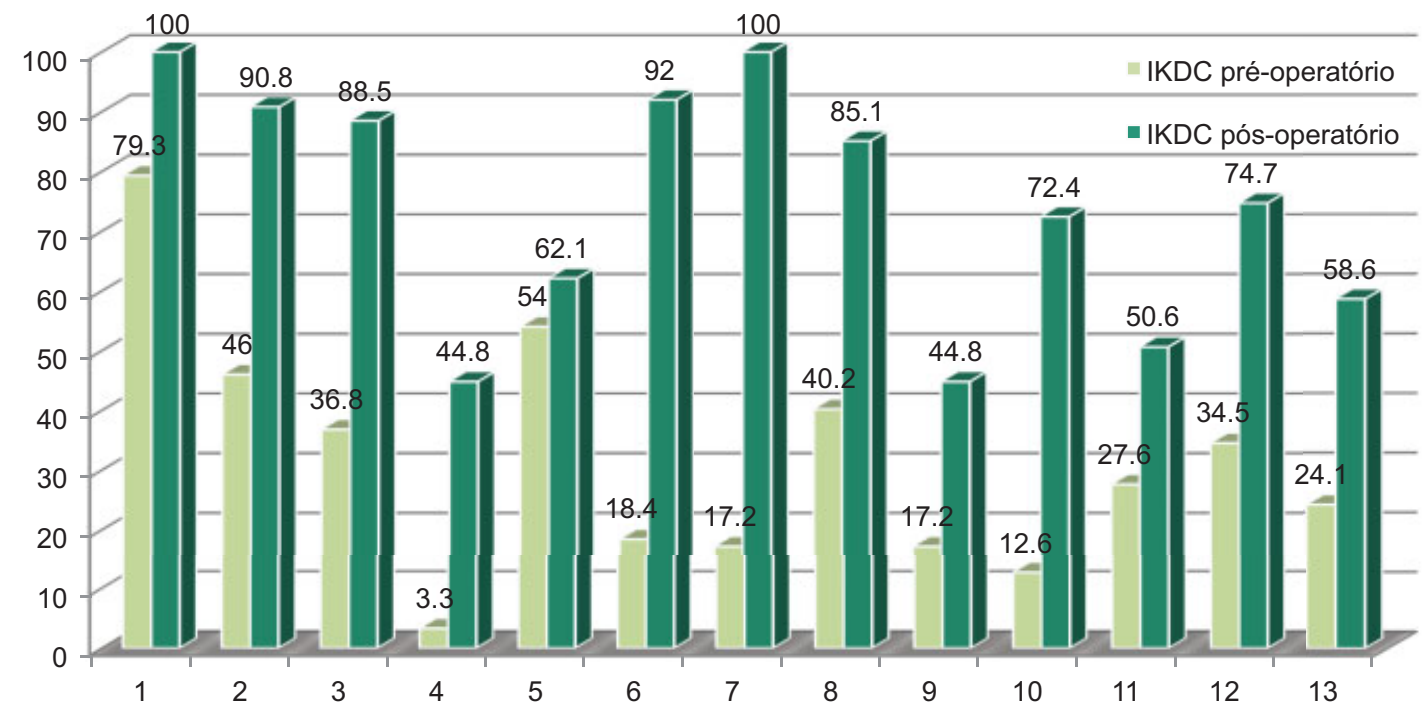

\begin{tabular}{|c|c|c|c|c|c|c|c|c|c|c|c|c|c|}
\hline Age & 25 & 16 & 16 & 17 & 54 & 18 & 36 & 30 & 42 & 48 & 56 & 31 & 53 \\
\hline Gender & M & F & F & M & F & M & F & F & F & F & F & M & F \\
\hline Localization & MFC & MFC & LFC & MFC & MFC & MFC & MFC & LFC & MFC & MFC & MFC & MFC & MFC \\
\hline Size & 1 & 3 & 4 & 2 & 1 & 3 & 2 & 1 & 0,6 & 2 & 0,9 & 1 & 2 \\
\hline$\triangle$ IKDC & $\mathbf{2 0 , 7}$ & $\mathbf{4 4 , 8}$ & $\mathbf{5 1 , 7}$ & $\mathbf{4 1 , 5}$ & $\mathbf{8 , 1}$ & $\mathbf{7 3 , 6}$ & $\mathbf{8 2 , 8}$ & $\mathbf{4 4 , 9}$ & $\mathbf{2 7 , 6}$ & $\mathbf{5 9 , 8}$ & $\mathbf{2 3 , 0}$ & $\mathbf{4 0 , 2}$ & $\mathbf{3 4 , 1}$ \\
\hline
\end{tabular}

Fig. 2 Resultados: o gráfico em barras mostra a pontuação do IKDC antes e após a cirurgia em cada paciente do estudo. Idade e acompanhamento em anos; Tamanho em $\mathrm{cm}^{2}$. Abreviaturas: F, feminino; IKDC, International Knee Documentation Committee; LFC, côndilo femoral lateral; M, masculino; MFC, côndilo femoral medial; - $\triangle$ IKDC = IKDC pós-operatório - IKDC pré-operatório.

artroplastia total do joelho (ATJ) 13 meses após a mosaicoplastia ( 6 anos após a revisão da ATJ). A pontuação média do IKDC no período pré-operatório foi de 31,63 pontos ( $\pm 20,24)$, enquanto a pontuação média no período pósoperatório foi de 74,18 pontos ( $\pm 20,26$ ). A diferença entre as pontuações do IKDC pós- e pré-operatórias foi de 42,55 pontos ( $\pm 21,05$ ), com aumento mínimo de 8,1 pontos, e aumento máximo de 82,8 pontos (-Fig. 2). Uma diferença estatisticamente significativa $(p<0,001)$ foi encontrada entre a pontuação IKDC antes e após a cirurgia no teste 


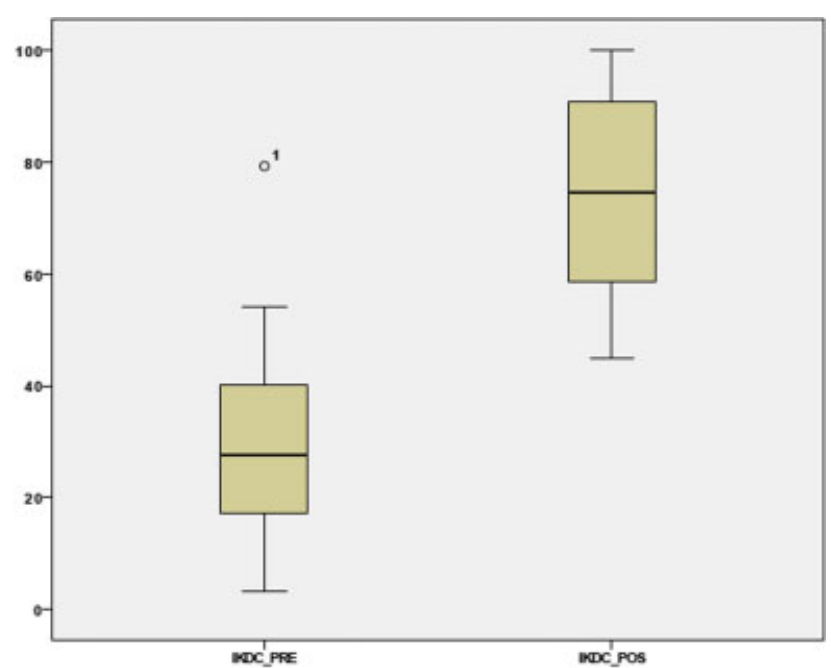

Fig. 3 Os gráficos em boxplot mostram as pontuações de IKDC antes e após a cirurgia, com análise estatística por teste não paramétrico de Wilcoxon de amostras pareadas, realizado no IBM SPSS Statistics for Windows, Versão 22.0) $-p<0,001$. Abreviações: IKDC, International Knee Documentation Committee.

de Wilcoxon com amostras pareadas (-Fig. 3). Correlações estatisticamente significativas entre a pontuação de IKDC e idade ou gênero não foram observadas. No entanto, houve uma relação estatisticamente significativa $(p=0,038)$ entre o aumento da pontuação do IKDC (a diferença entre a pontuação pós-operatória e pré-operatória) e a dimensão da lesão.

\section{Discussão}

O tratamento das lesões da cartilagem articular inlcui procedimentos paliativos (desbridamento e condroplastia), reparadores (microfratura) e reconstrutivos (ACI, OTA, e OCA). ${ }^{2,4,10}$ A sua aplicação é orientada por critérios clínicos e morfológicos. De acordo com as mais recentes revisões da literatura que discutem o tratamento das lesões osteocondrais do joelho, aquelas com $<2 \mathrm{~cm}^{2}$ são melhor tratadas por microfratura (opção de primeira linha) ou por OTA. ${ }^{11}$ Esta última técnica apresenta resultados de maior longevidade e durabilidade, em especial entre pacientes com alta demanda funcional, em comparação à microfratura. ${ }^{3,4}$ As lesões com dimensões entre $2 \mathrm{e}$ $4 \mathrm{~cm}^{2}$ devem ser tratadas por OTA ou ACI. ${ }^{3}$ Em relação às lesões com dimensão $\geq 4 \mathrm{~cm}^{2}$, a morbidade da OTA não é negligenciável e, por isso, o tratamento deve ser feito por ACI ou por OCA (sendo esta última aplicada especialmente em lesões pós-traumáticas extensas ou em casos de osteocondrite dissecante). ${ }^{3}$ A OTA, como outras técnicas reconstrutivas, tem bons resultados sintomáticos e funcionais e previne a progressão das alterações degenerativas. Além disso, apresenta algumas vantagens, como baixo custo, ausência de reações imunes e transmissão de doenças infeciosas (em comparação ao OCA) e pode ser feita em um único tempo cirúrgico. ${ }^{2,3,7-9}$ Este procedimento, quando realizado em lesões com dimensões entre 2 e 4 $\mathrm{cm}^{2}$, tem maior eficácia e durabilidade; como demons- trado no nosso estudo (que analisou lesões até estas dimensões), é possível estabelecer uma correlação de significado estatístico entre o ganho de IKDC e o tamanho da lesão. A taxa de complicações conhecidas é baixa, como observado no nosso grupo de estudo. Os resultados tendem a ser bons no médio e longo prazo, sendo piores com o aumento da idade, sexo feminino e defeitos maiores ( $>4$ $\left.\mathrm{cm}^{2}\right)^{2,3}$ A melhora significativa do IKDC observada no nosso estudo ao compararmos o estado pré- e pós-operatório condiz com outros estudos recentes que usam esta ou outras pontuações de avaliação funcional. ${ }^{9,12,13}$

\section{Conclusão}

A regeneração da cartilagem hialina ainda não é possível e continua a ser o maior objetivo a alcançar no tratamento de lesões osteocondrais. A mosaicoplastia com OTA, quando adequadamente utilizada, pode produzir excelentes resultados com grande durabilidade e impacto funcional, além de baixas taxas de morbidade e baixos custos. Embora tenha indicações restritas, a expansão dos critérios para o seu uso teve resultados promissores no médio e longo prazo, como mostra a literatura recente.

Conflitos de Interesses

Os autores declaram não haver conflitos de interesses.

\section{Referências}

1 Curl WW, Krome J, Gordon ES, Rushing J, Smith BP, Poehling GG. Cartilage injuries: a review of 31,516 knee arthroscopies. Arthroscopy 1997;13(04):456-460

2 Berta Á, Duska Z, Tóth F, Hangody L. Clinical experiences with cartilage repair techniques: outcomes, indications, contraindications and rehabilitation. Eklem Hastalik Cerrahisi 2015;26(02): 84-96

3 Krych AJ, Harnly HW, Rodeo SA, Williams RJ III. Activity levels are higher after osteochondral autograft transfer mosaicplasty than after microfracture for articular cartilage defects of the knee: a retrospective comparative study. J Bone Joint Surg Am 2012;94 (11):971-978

4 Gudas R, Kalesinskas RJ, Kimtys V, Stankevicius E, Toliusis V, Bernotavicius $\mathrm{G}$, et al. A prospective randomized clinical study of mosaic osteochondral autologous transplantation versus microfracture for the treatment of osteochondral defects in the knee joint in young athletes. Arthroscopy 2005;21(09): 1066-1075

5 Smith GD, Knutsen G, Richardson JB. A clinical review of cartilage repair techniques. J Bone Joint Surg Br 2005;87(04):445-449

6 Bedi A, Feeley BT, Williams RJ III. Management of articular cartilage defects of the knee. J Bone Joint Surg Am 2010;92(04): 994-1009

7 Hangody L, Kish G, Kárpáti Z, Szerb I, Udvarhelyi I. Arthroscopic autogenous osteochondral mosaicplasty for the treatment of femoral condylar articular defects. A preliminary report. Knee Surg Sports Traumatol Arthrosc 1997;5(04):262-267

8 Hangody L, Füles P. Autologous osteochondral mosaicplasty for the treatment of full-thickness defects of weight-bearing joints: ten years of experimental and clinical experience. J Bone Joint Surg Am 200385-A(Suppl 2):25-32

9 Hangody L, Dobos J, Baló E, Pánics G, Hangody LR, Berkes I. Clinical experiences with autologous osteochondral mosaicplasty in an 
athletic population: a 17-year prospective multicenter study. Am J Sports Med 2010;38(06):1125-1133

10 McNickle AG, Provencher MT, Cole BJ. Overview of existing cartilage repair technology. Sports Med Arthrosc Rev 2008;16(04):196-201

11 Richter DL, Schenck RC Jr, Wascher DC, Treme G. Knee Articular Cartilage Repair and Restoration Techniques: A Review of the Literature. Sports Health 2016;8(02):153-160
12 Solheim E, Hegna J, Øyen J, Harlem T, Strand T. Results at 10 to 14 years after osteochondral autografting (mosaicplasty) in articular cartilage defects in the knee. Knee 2013;20(04):287-290

13 Reverte-Vinaixa MM, Joshi N, Diaz-Ferreiro EW, Teixidor-Serra J, Dominguez-Oronoz R. Medium-term outcome of mosaicplasty for grade III-IV cartilage defects of the knee. J Orthop Surg (Hong Kong) $2013 ; 21(01): 4-9$ 